\title{
In silico evaluation of the radical scavenging mechanism of Mactanamide
}

\author{
Quan V. Vo ${ }^{1,2^{*}}$, Nguyen Thi Hoa ${ }^{2}$, Pham Cam Nam ${ }^{3 *}$, Duong Tuan Quang ${ }^{4}$ and Adam \\ Mechler $^{5 *}$
}

${ }^{1}$ Institute of Research and Development, Duy Tan University, Danang 550000, Vietnam

${ }^{2}$ The University of Danang - University of Technology and Education, 48 Cao Thang, Danang 550000 , Vietnam.

${ }^{3}$ Department of Chemical Engineering, The University of Danang - University of Science and Technology, Danang 550000, Vietnam.

${ }^{4}$ University of Education, Hue University, Hue City 530000, Vietnam.

${ }^{5}$ Department of Chemistry and Physics, La Trobe University, Victoria 3086, Australia

*Corresponding authors: vovanquan2@duytan.edu.vn, vvquan@ute.udn.vn; pcnam@dut.udn.vn, a.mechler@ltu.edu.au

\section{Table of Contents}

Table S1. The method to calculate rate constant following the conventional transition state theory .......

Table S2: The Cartesian coordinates and energies of pre-complexes, TS and post-complexes of the reaction between $\mathrm{M}$ with $\mathrm{HOO}^{\circ}$ at the $\mathrm{M06}-2 \mathrm{X} / 6-311++\mathrm{G}(\mathrm{d}, \mathrm{p})$ calculating method following the FHT mechanism. 
Table S1. The method to calculate rate constant following the conventional transition state theory

The rate constant $(k)$ was calculated by using the conventional transition state theory (TST) (at $298.15 \mathrm{~K}, 1 \mathrm{M}$ standard state) according to the equation (1): $:^{1-5}$

$k=\sigma \kappa \frac{k_{B} T}{h} e^{-\left(\Delta G^{\ddagger}\right) / R T}$

Where: $\quad \sigma$ is the reaction symmetry number $, 6,7$

$\kappa$ contains the tunneling corrections calculated using the Eckart barrier, ${ }^{8}$

$k_{B}$ is the Boltzmann constant,

$h$ is the Planck constant,

$\Delta \mathrm{G}^{\neq}$is the Gibbs free energy of activation.

The Marcus Theory was used to estimate the reaction barriers of SET reactions. ${ }^{9-12}$ The free energy of reaction $\Delta G^{\neq}$for the SET pathway was computed following the equations $(2,3)$.

$\Delta \mathrm{G}_{\mathrm{SET}}^{\neq}=\frac{\lambda}{4}\left(1+\frac{\Delta G_{S E T}^{0}}{\lambda}\right)^{2}$
$\lambda \approx \Delta \mathrm{E}_{\mathrm{SET}}-\Delta \mathrm{G}_{\mathrm{SET}}^{0}$

where $\Delta \mathrm{G}_{\mathrm{SET}}$ is the Gibbs energy of reaction, $\Delta \mathrm{E}_{\mathrm{SET}}$ is the non-adiabatic energy difference between reactants and vertical products for SET. ${ }^{13,14}$

For rate constants that were close to the diffusion limit a correction was applied to yield realistic results ${ }^{15}$. The apparent rate constants $\left(k_{\mathrm{app}}\right)$ were calculated following the Collins-Kimball theory in the solvents at $298.15 \mathrm{~K} ;{ }^{16}$ the steady-state Smoluchowski rate constant $\left(k_{\mathrm{D}}\right)$ for an irreversible bimolecular diffusion-controlled reaction was calculated following the literature as corroding to equations $(4,5) \cdot{ }^{15,17}$

$$
\begin{aligned}
& k_{\mathrm{app}}=\frac{\mathrm{k}_{\mathrm{TST}} \mathrm{k}_{\mathrm{D}}}{\mathrm{k}_{\mathrm{TST}}+\mathrm{k}_{\mathrm{D}}} \\
& k_{\mathrm{D}}=4 \pi R_{A B} D_{A B} N_{A}
\end{aligned}
$$

where $R_{A B}$ is the reaction distance, $N_{A}$ is the Avogadro constant, and $D_{A B}=D_{A}+D_{B}\left(D_{A B}\right.$ is the mutual diffusion coefficient of the reactants $A$ and $B),{ }^{16,18}$ where $D_{A}$ or $D_{B}$ is estimated using the Stokes-Einstein formulation (6). ${ }^{19,20}$

$$
D_{A \text { or } B}=\frac{k_{\mathrm{B}} T}{6 \pi \eta a_{A \text { or } B}}
$$

$\eta$ is the viscosity of the solvents (i.e. $\eta\left(\mathrm{H}_{2} \mathrm{O}\right)=8.91 \times 10^{-4}$ Pa s, $\eta$ (pentyl ethanoate) $=8.62 \times 10^{-4} \mathrm{~Pa} \mathrm{~s}$ ) and $a$ is the radius of the solute.

The kinetic study requires different considerations. Water (dielectric constants, $\varepsilon=78.35$ ) and pentyl ethanoate $(\varepsilon=4.73)$ are the de facto standard solvents in the literature to mimic the polar and nonpolar environments in the human body. ${ }^{15,21-23}$ Thus, these solvents were used to model the physiological environments. The solvent cage effects were included following the corrections proposed by Okuno, ${ }^{24}$ adjusted with the free volume theory according to the Benson correction ${ }^{15,25-27}$ to reduce over-penalizing entropy losses in solution. For the species that have multiple conformers, all of these were investigated and the conformer with the lowest electronic energy was included in the analysis. $^{22,23}$ The hindered internal rotation treatment was also applied to the single bonds to ensure that the obtained conformer has the lowest electronic energy. ${ }^{23,28}$ All transition states were characterized by the existence of only one single imaginary frequency. Intrinsic coordinate calculations (IRCS) were performed to ensure that each transition state is connected correctly with the pre-complex and post-complex. 
Table S2: The Cartesian coordinates and energies of pre-complexes, TS and post-complexes of the reaction between $\mathrm{M}$ with $\mathrm{HOO}^{\circ}$ at the M06-2X/6-311++G(d,p) calculating method following the FHT mechanism.

\begin{tabular}{|c|c|c|c|c|c|}
\hline \multicolumn{4}{|l|}{ Name } & \multicolumn{2}{|l|}{ Pre-complexes-M-C3-OOH(gas) } \\
\hline \multicolumn{4}{|c|}{ Cartesian Coordinates } & Frequency and Energy & \\
\hline $\mathrm{N}$ & -1.92951500 & -1.22255600 & 1.59967300 & Zero-point correction $=$ & (Hartree/Particle) \\
\hline C & -0.74457400 & -0.61231600 & 1.40319400 & Thermal correction to Energy= & 2138 \\
\hline C & 0.00991300 & -0.87576900 & 0.11490000 & Thermal correction to Enthalpy= & 13082 \\
\hline $\mathrm{N}$ & -0.70705000 & -1.72284000 & -0.81603300 & Thermal correction to Gibbs Free Energy= & 0.330221 \\
\hline $\mathrm{C}$ & -1.99818000 & -2.10190700 & -0.74503700 & Sum of electronic and zero-point Energies= & -1296.896828 \\
\hline $\mathrm{C}$ & -2.76880900 & -1.69734300 & 0.50421300 & Sum of electronic and thermal Energies= & -1296.871730 \\
\hline $\mathrm{C}$ & -2.57129700 & -1.03912600 & 2.89618900 & Sum of electronic and thermal Enthalpies= & -1296.870786 \\
\hline $\mathrm{O}$ & -0.22528300 & 0.10205100 & 2.25631200 & Sum of electronic and thermal Free Energies= & -1296.953647 \\
\hline C & 0.45617400 & 0.47020300 & -0.51391900 & & \\
\hline C & 1.90171500 & 0.77871800 & -0.23920800 & & \\
\hline $\mathrm{C}$ & 2.32734300 & 1.64290300 & 0.77744100 & & \\
\hline $\mathrm{C}$ & 3.68522800 & 1.93819300 & 0.93571000 & & \\
\hline $\mathrm{C}$ & 4.62150700 & 1.33843900 & 0.10973800 & & \\
\hline $\mathrm{C}$ & 4.23038000 & 0.42352100 & -0.86989800 & & \\
\hline C & 2.87281400 & 0.14581600 & -1.02061400 & & \\
\hline 0 & -2.56946000 & -2.71577400 & -1.62478900 & & \\
\hline $\mathrm{C}$ & -3.83827900 & -0.65185600 & 0.10405700 & & \\
\hline $\mathrm{C}$ & -3.22755100 & 0.62062700 & -0.43028600 & & \\
\hline $\mathrm{C}$ & -2.94205000 & 1.69193200 & 0.41849900 & & \\
\hline $\mathrm{C}$ & -2.36329900 & 2.85848400 & -0.07300000 & & \\
\hline $\mathrm{C}$ & -2.06101700 & 2.96710300 & -1.42647900 & & \\
\hline $\mathrm{C}$ & -2.33399800 & 1.90297700 & -2.28108700 & & \\
\hline $\mathrm{C}$ & -2.91064900 & 0.73767700 & -1.78663600 & & \\
\hline 0 & 1.45122000 & 2.22287300 & 1.62752000 & & \\
\hline 0 & 2.41386900 & -0.76492100 & -1.93088500 & & \\
\hline $\mathrm{H}$ & -4.46232400 & -0.43640000 & 0.97522000 & & \\
\hline $\mathrm{H}$ & -4.47155000 & -1.12017200 & -0.65190000 & & \\
\hline $\mathrm{H}$ & -3.18183900 & 1.61975000 & 1.47456600 & & \\
\hline $\mathrm{H}$ & -3.12455100 & -0.09130500 & -2.45490600 & & \\
\hline $\mathrm{H}$ & -2.14720200 & 3.67874400 & 0.60125200 & & \\
\hline $\mathrm{H}$ & -2.10141600 & 1.98114400 & -3.33677900 & & \\
\hline $\mathrm{H}$ & -1.61338300 & 3.87514700 & -1.81246700 & & \\
\hline $\mathrm{H}$ & -3.27871800 & -2.60427300 & 0.83956600 & & \\
\hline $\mathrm{H}$ & 0.90859600 & -1.41618100 & 0.43444900 & & \\
\hline $\mathrm{H}$ & -0.21967500 & -1.97557400 & -1.66754000 & & \\
\hline $\mathrm{H}$ & -3.34540600 & -1.79855500 & 3.00891600 & & \\
\hline $\mathrm{H}$ & -1.82545600 & -1.15179900 & 3.68032900 & & \\
\hline $\mathrm{H}$ & -3.01980400 & -0.04668600 & 2.99117300 & & \\
\hline $\mathrm{H}$ & -0.18412300 & 1.28070600 & -0.16294500 & & \\
\hline $\mathrm{H}$ & 0.30960200 & 0.39461200 & -1.59190700 & & \\
\hline $\mathrm{H}$ & 3.97482100 & 2.61922300 & 1.72515400 & & \\
\hline $\mathrm{H}$ & 5.67338000 & 1.56749300 & 0.23318700 & & \\
\hline $\mathrm{H}$ & 4.95825100 & -0.05606400 & -1.51489700 & & \\
\hline $\mathrm{H}$ & 0.75883600 & 1.57685200 & 1.86359900 & & \\
\hline $\mathrm{H}$ & 3.13061100 & -1.37269100 & -2.14697700 & & \\
\hline $\mathrm{H}$ & 4.11968300 & -1.20213500 & 0.60666100 & & \\
\hline 0 & 4.07246400 & -2.65716200 & -0.51048000 & & \\
\hline 0 & 4.04786400 & -2.17878300 & 0.70158400 & & \\
\hline Name & & & & TS-M-C3-OOH(gas) & \\
\hline
\end{tabular}




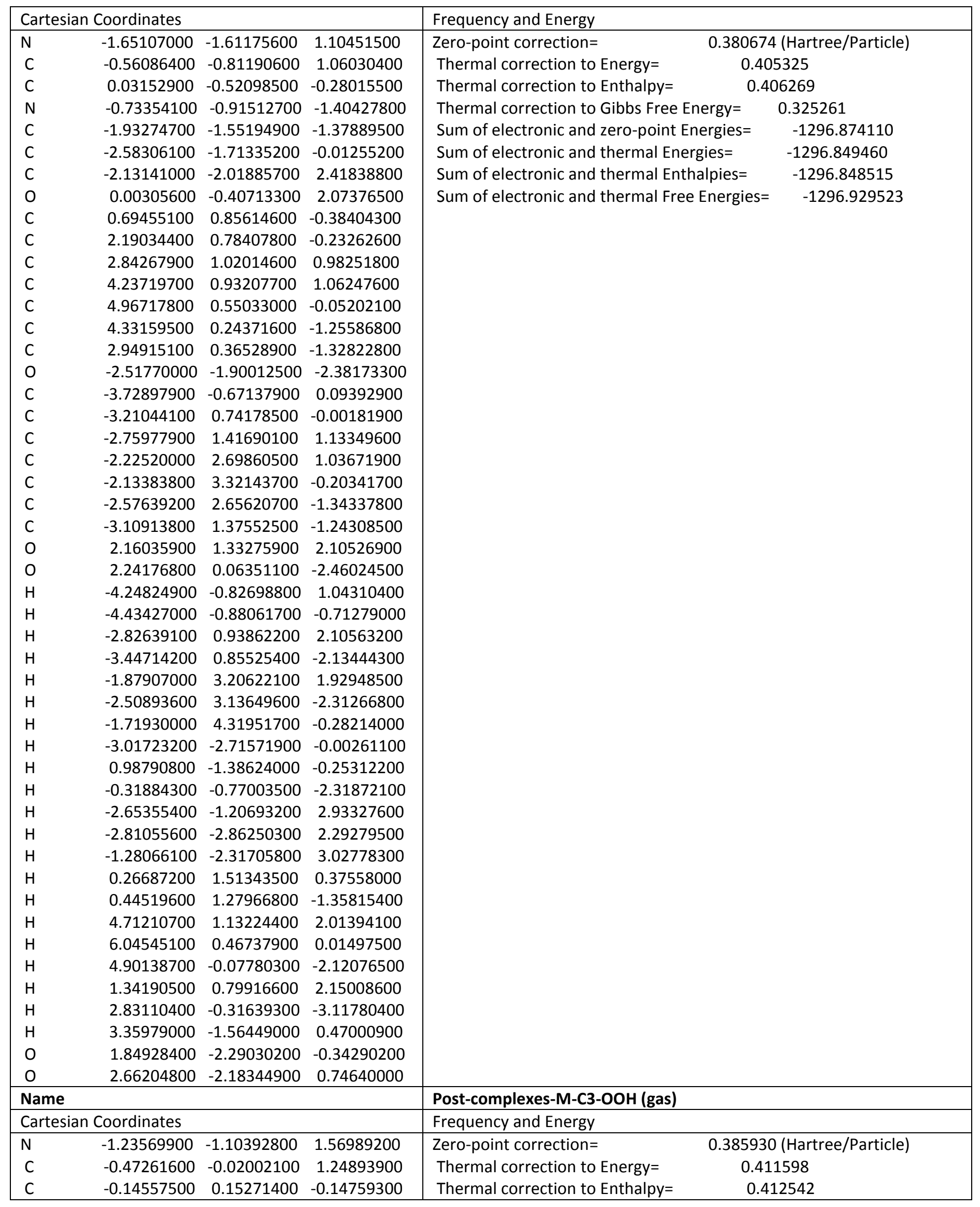




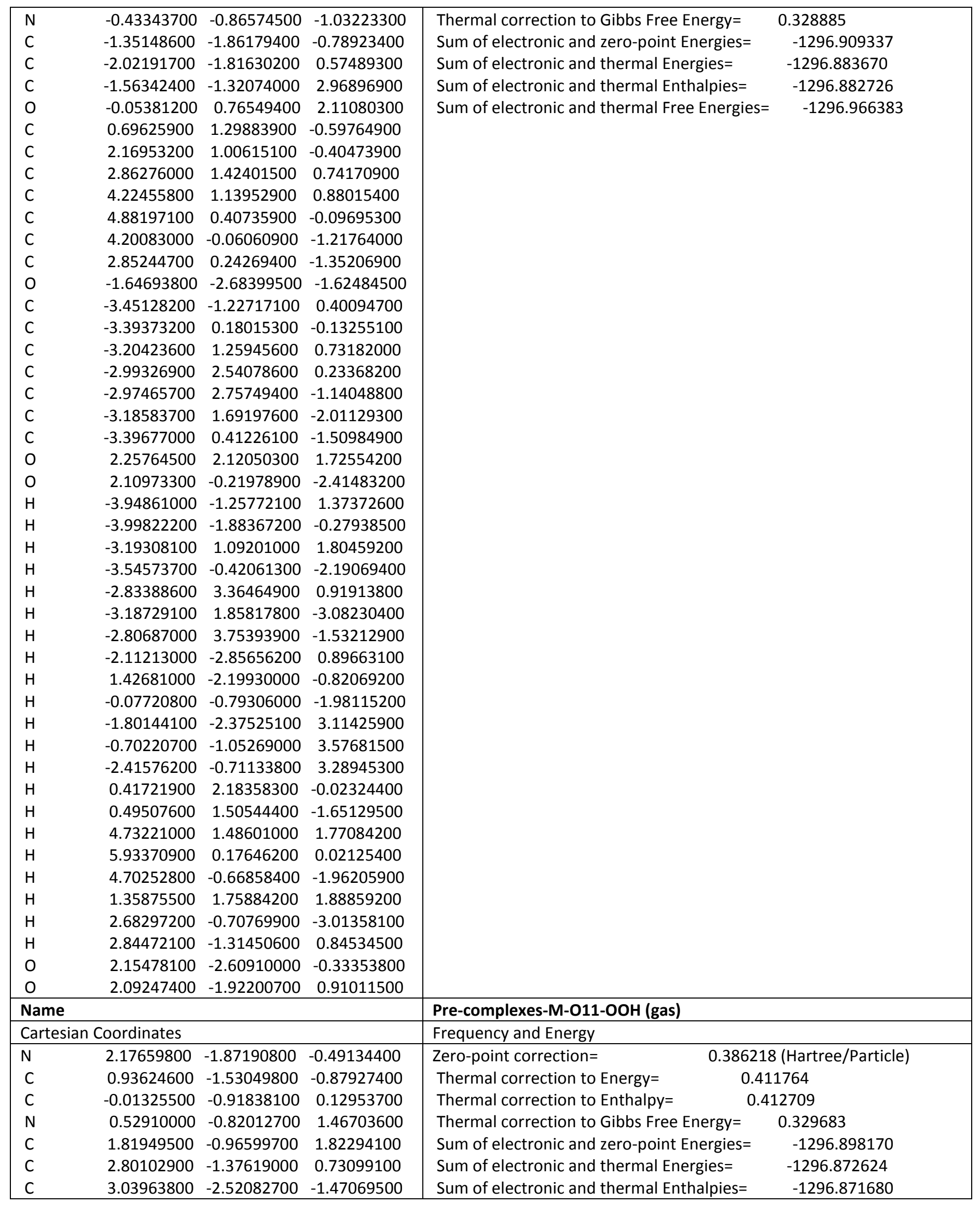




\begin{tabular}{|c|c|c|c|c|c|}
\hline $\mathrm{O}$ & 0.51045100 & -1.73321900 & -2.01404600 & \multirow[t]{41}{*}{ Sum of electronic and thermal Free Energies= } & \multirow{41}{*}{-1296.954706} \\
\hline $\mathrm{C}$ & -0.54536700 & 0.43835100 & -0.41856900 & & \\
\hline $\mathrm{C}$ & -2.04529100 & 0.48796600 & -0.45316500 & & \\
\hline $\mathrm{C}$ & -2.75918200 & -0.20632200 & -1.44076600 & & \\
\hline C & -4.16019600 & -0.18034300 & -1.45751400 & & \\
\hline C & -4.84461700 & 0.51983300 & -0.47877800 & & \\
\hline C & -4.15762700 & 1.19267800 & 0.53473100 & & \\
\hline $\mathrm{C}$ & -2.76387200 & 1.17425500 & 0.53070200 & & \\
\hline $\mathrm{O}$ & 2.23899700 & -0.76732800 & 2.94647700 & & \\
\hline $\mathrm{C}$ & 3.75479100 & -0.19049000 & 0.45645600 & & \\
\hline $\mathrm{C}$ & 3.02361600 & 1.03912800 & -0.02031300 & & \\
\hline $\mathrm{C}$ & 2.77099000 & 1.24442700 & -1.37760500 & & \\
\hline $\mathrm{C}$ & 2.08711600 & 2.37648600 & -1.81164300 & & \\
\hline $\mathrm{C}$ & 1.64226000 & 3.31677400 & -0.88873100 & & \\
\hline $\mathrm{C}$ & 1.87950700 & 3.11713400 & 0.46863400 & & \\
\hline $\mathrm{C}$ & 2.56283200 & 1.98546300 & 0.89990400 & & \\
\hline $\mathrm{O}$ & -2.15827700 & -0.92023200 & -2.40946600 & & \\
\hline 0 & -2.03139800 & 1.80628400 & 1.49057300 & & \\
\hline $\mathrm{H}$ & 4.49796400 & -0.50429100 & -0.28134600 & & \\
\hline $\mathrm{H}$ & 4.27863700 & 0.01733600 & 1.39111400 & & \\
\hline $\mathrm{H}$ & 3.12338300 & 0.51923000 & -2.10495500 & & \\
\hline $\mathrm{H}$ & 2.74266100 & 1.82624800 & 1.95869100 & & \\
\hline $\mathrm{H}$ & 1.90394900 & 2.52279700 & -2.86979800 & & \\
\hline $\mathrm{H}$ & 1.52977100 & 3.84312300 & 1.19331200 & & \\
\hline $\mathrm{H}$ & 1.11155100 & 4.20000100 & -1.22394200 & & \\
\hline $\mathrm{H}$ & 3.38482600 & -2.19503500 & 1.16024200 & & \\
\hline $\mathrm{H}$ & -0.84522900 & -1.63135100 & 0.16266800 & & \\
\hline $\mathrm{H}$ & -0.10916200 & -0.53717200 & 2.20221300 & & \\
\hline $\mathrm{H}$ & 3.47618800 & -1.79791000 & -2.16574000 & & \\
\hline $\mathrm{H}$ & 3.83941400 & -3.03943500 & -0.94133000 & & \\
\hline $\mathrm{H}$ & 2.45130700 & -3.23348200 & -2.04515300 & & \\
\hline $\mathrm{H}$ & -0.15650200 & 0.59333900 & -1.42729200 & & \\
\hline $\mathrm{H}$ & -0.14917600 & 1.23987100 & 0.20271700 & & \\
\hline $\mathrm{H}$ & -4.67661300 & -0.72106000 & -2.23969600 & & \\
\hline $\mathrm{H}$ & -5.92775900 & 0.53792000 & -0.48902000 & & \\
\hline $\mathrm{H}$ & -4.69481300 & 1.73575200 & 1.30459600 & & \\
\hline $\mathrm{H}$ & -1.20867300 & -1.10281600 & -2.26179600 & & \\
\hline $\mathrm{H}$ & -2.61926700 & 2.19361000 & 2.14460400 & & \\
\hline $\mathrm{H}$ & -3.75331400 & -1.04199000 & 1.02105100 & & \\
\hline 0 & -2.34871700 & -1.38839400 & 2.15728200 & & \\
\hline 0 & -3.37451900 & -1.82225000 & 1.48142600 & & \\
\hline \multicolumn{4}{|c|}{ Name } & \multicolumn{2}{|l|}{ TS-M-O11-OOH (gas) } \\
\hline \multicolumn{4}{|c|}{ Cartesian Coordinates } & \multicolumn{2}{|l|}{ Frequency and Energy } \\
\hline $\mathrm{N}$ & 2.26761300 & 1.39101100 & -1.23314000 & \multicolumn{2}{|c|}{0.381506 (Hartree/Particle) } \\
\hline $\mathrm{C}$ & 0.98803000 & 1.55000900 & -0.86062400 & \multicolumn{2}{|l|}{ Thermal correction to Energy= } \\
\hline $\mathrm{C}$ & 0.02743500 & 0.38195600 & -0.96897100 & \multicolumn{2}{|l|}{ Thermal correction to Enthalpy= } \\
\hline $\mathrm{N}$ & 0.62446100 & -0.80553800 & -1.54460100 & \multicolumn{2}{|l|}{ Thermal correction to Gibbs Free Energy= } \\
\hline $\mathrm{C}$ & 1.94002200 & -1.04705900 & -1.70384200 & \multicolumn{2}{|l|}{ Sum of electronic and zero-point Energies= } \\
\hline $\mathrm{C}$ & 2.90479100 & 0.08745900 & -1.38427300 & Sum of electronic and thermal Energies= & \multirow{2}{*}{$\begin{array}{l}-1296.855803 \\
-1296854859\end{array}$} \\
\hline $\mathrm{C}$ & 3.13705300 & 2.56071700 & -1.18319700 & \multirow{5}{*}{$\begin{array}{l}\text { Sum of electronic and thermal Enthalpies= } \\
\text { Sum of electronic and thermal Free Energies= }\end{array}$} & \\
\hline $\mathrm{O}$ & 0.54697200 & 2.63180400 & -0.47376600 & & -1296.935013 \\
\hline $\mathrm{C}$ & -0.64524800 & 0.12956400 & 0.41913800 & & \\
\hline $\mathrm{C}$ & -2.13646600 & 0.30949300 & 0.41909700 & & \\
\hline $\mathrm{C}$ & -2.72308400 & 1.57733400 & 0.37772300 & & \\
\hline
\end{tabular}




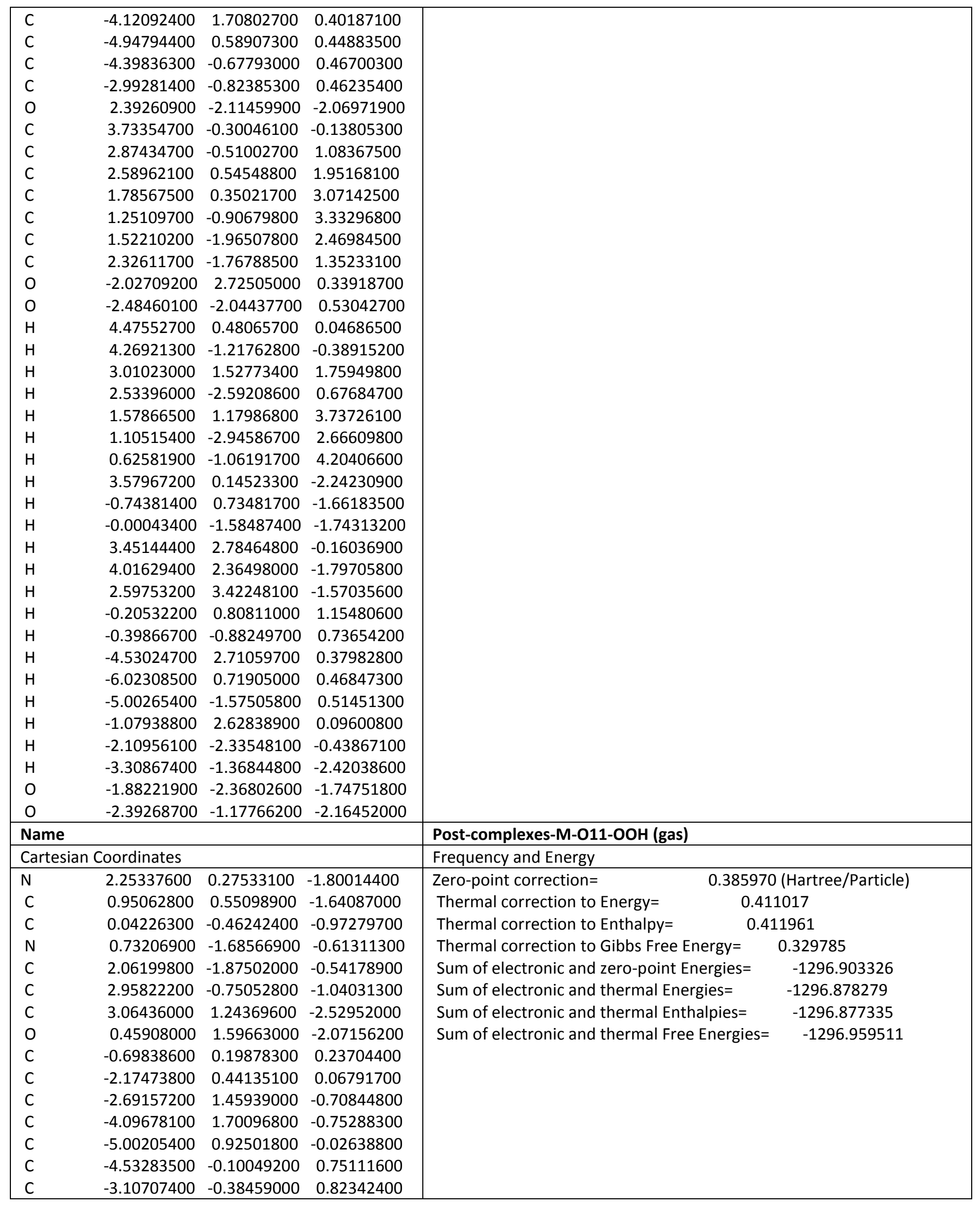




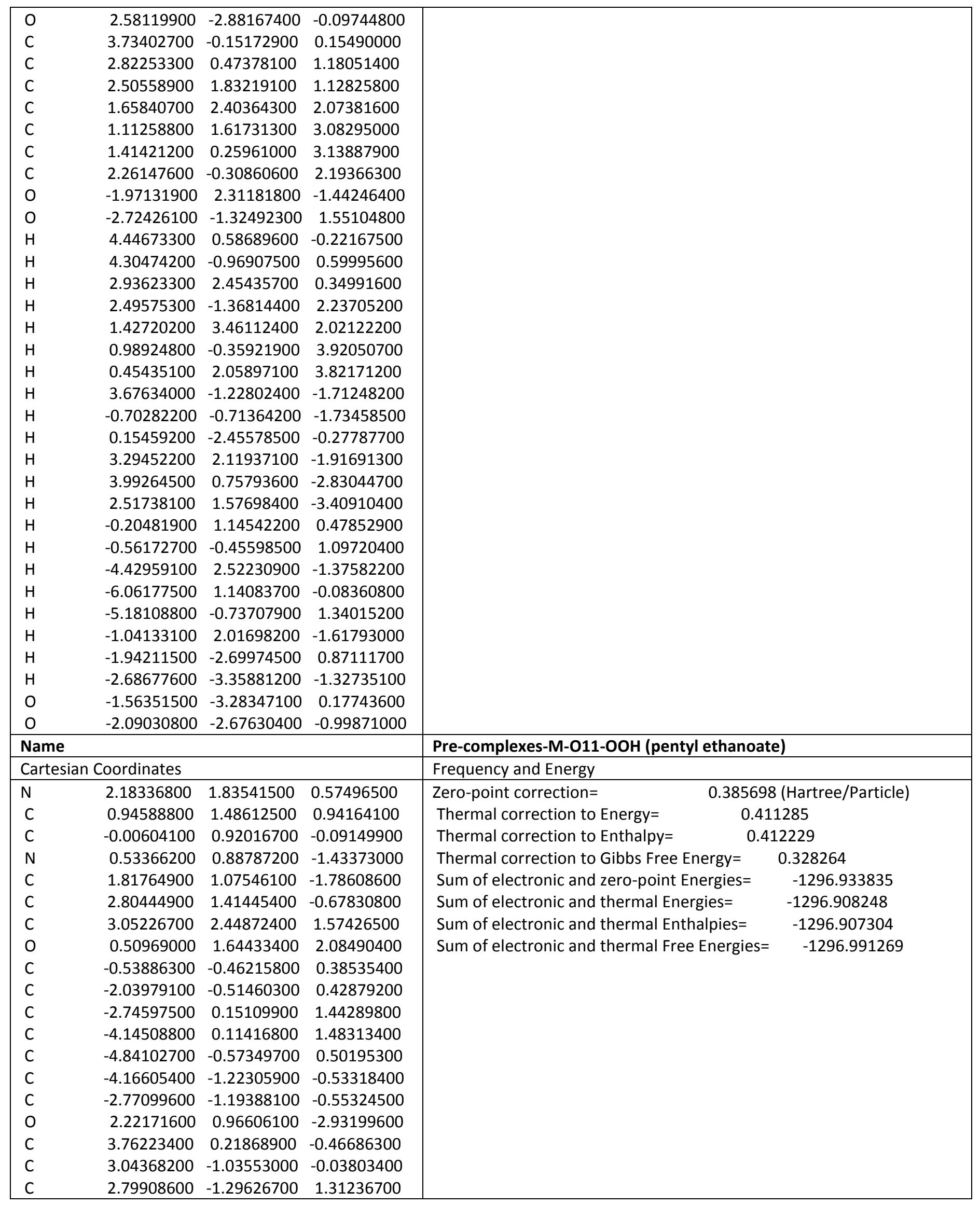




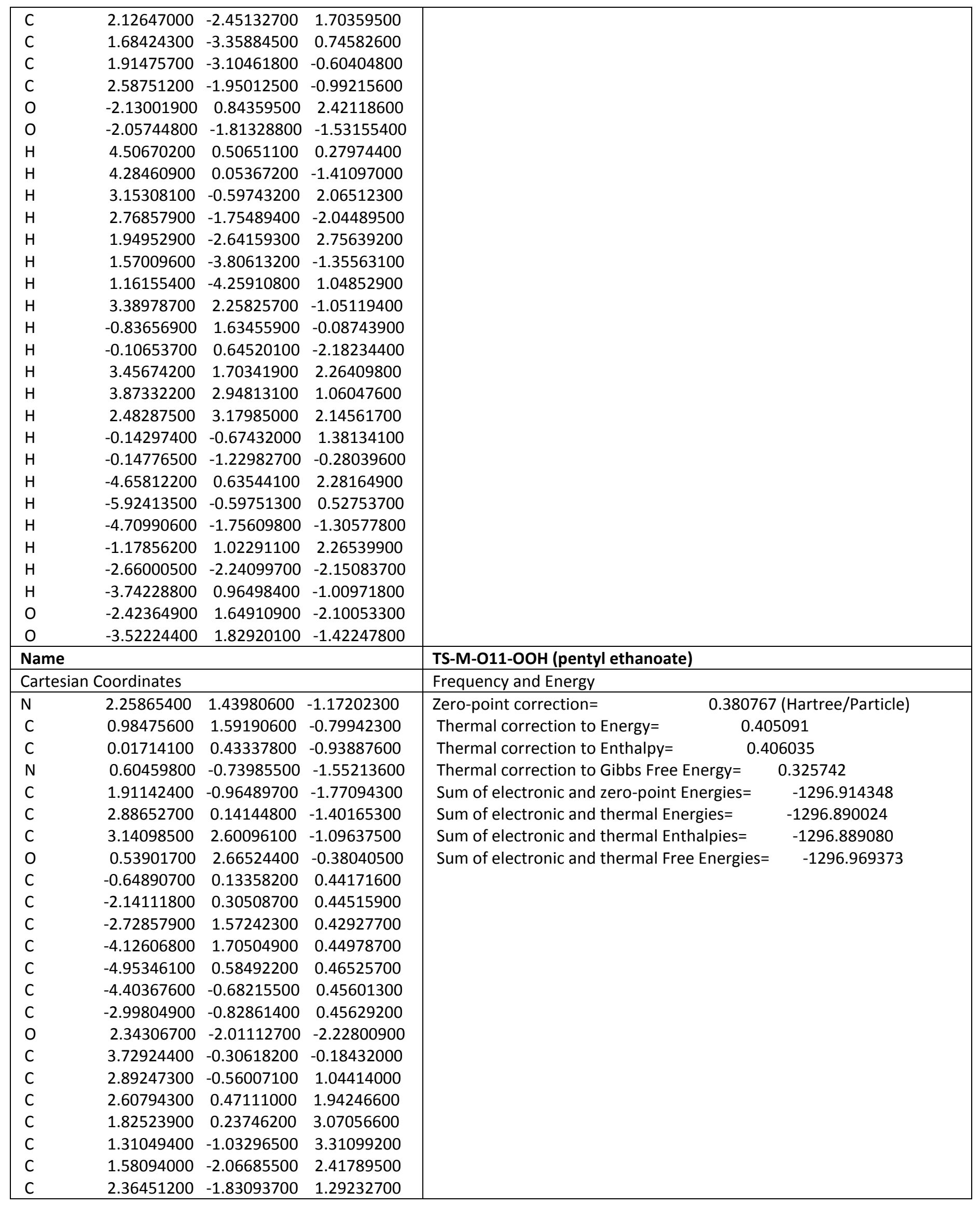




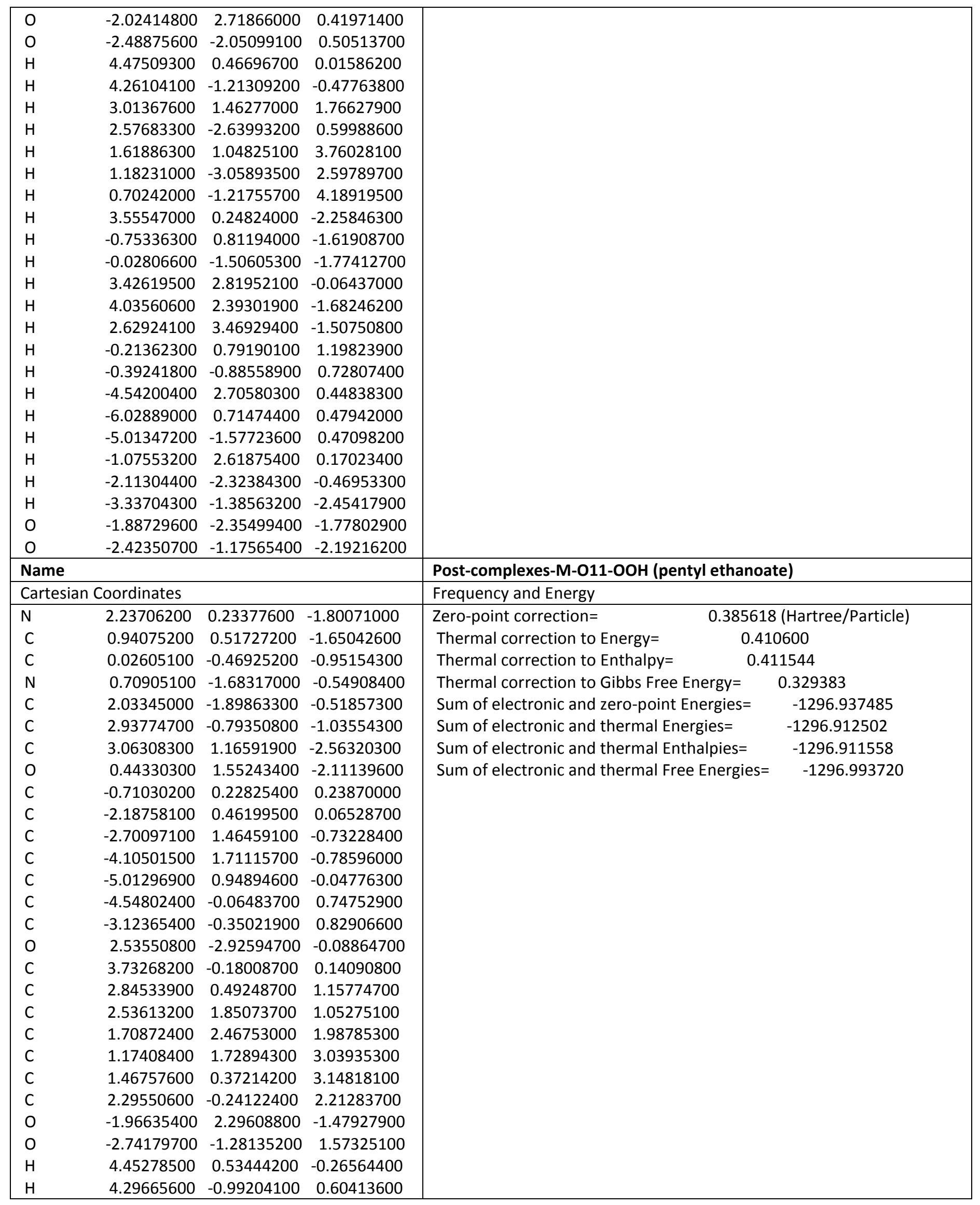




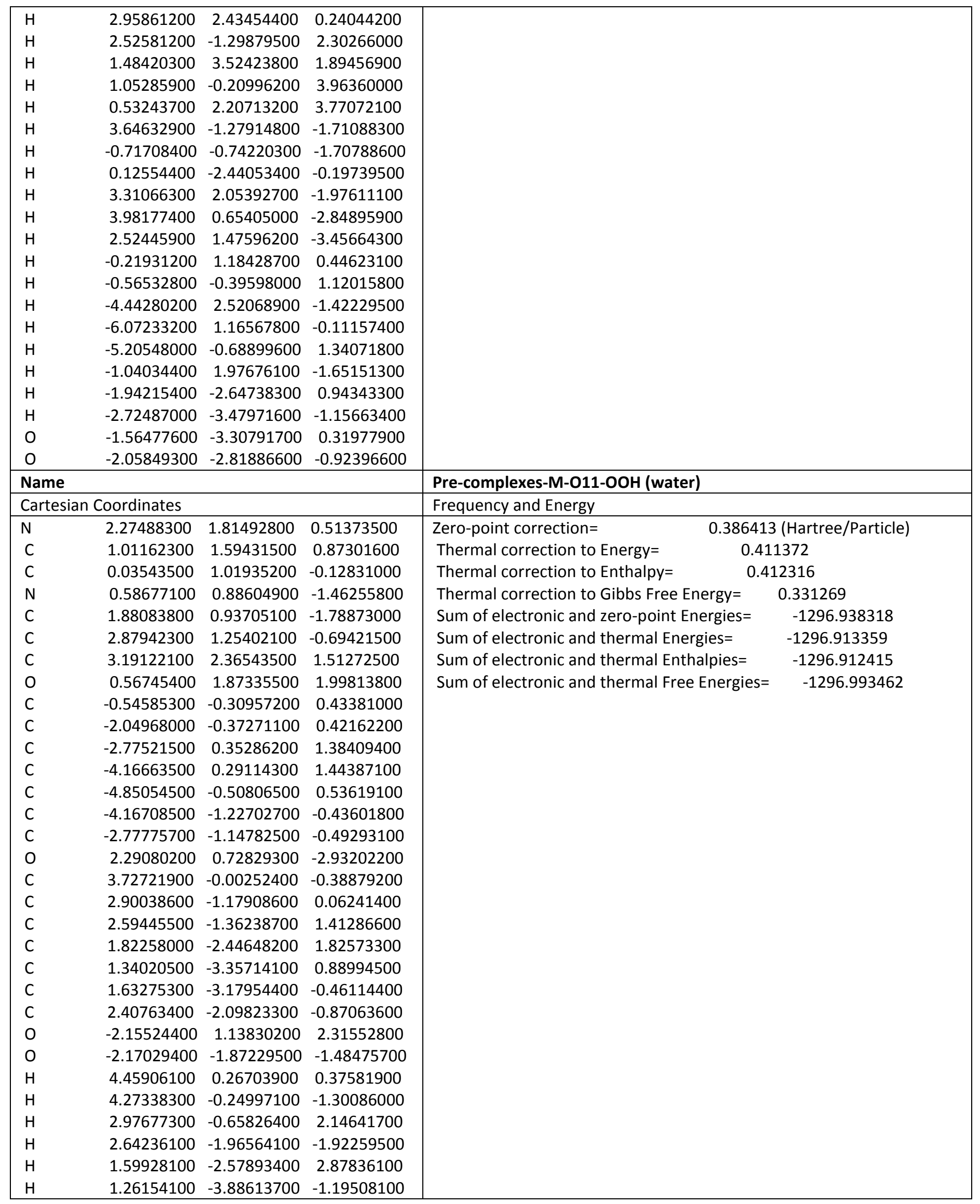




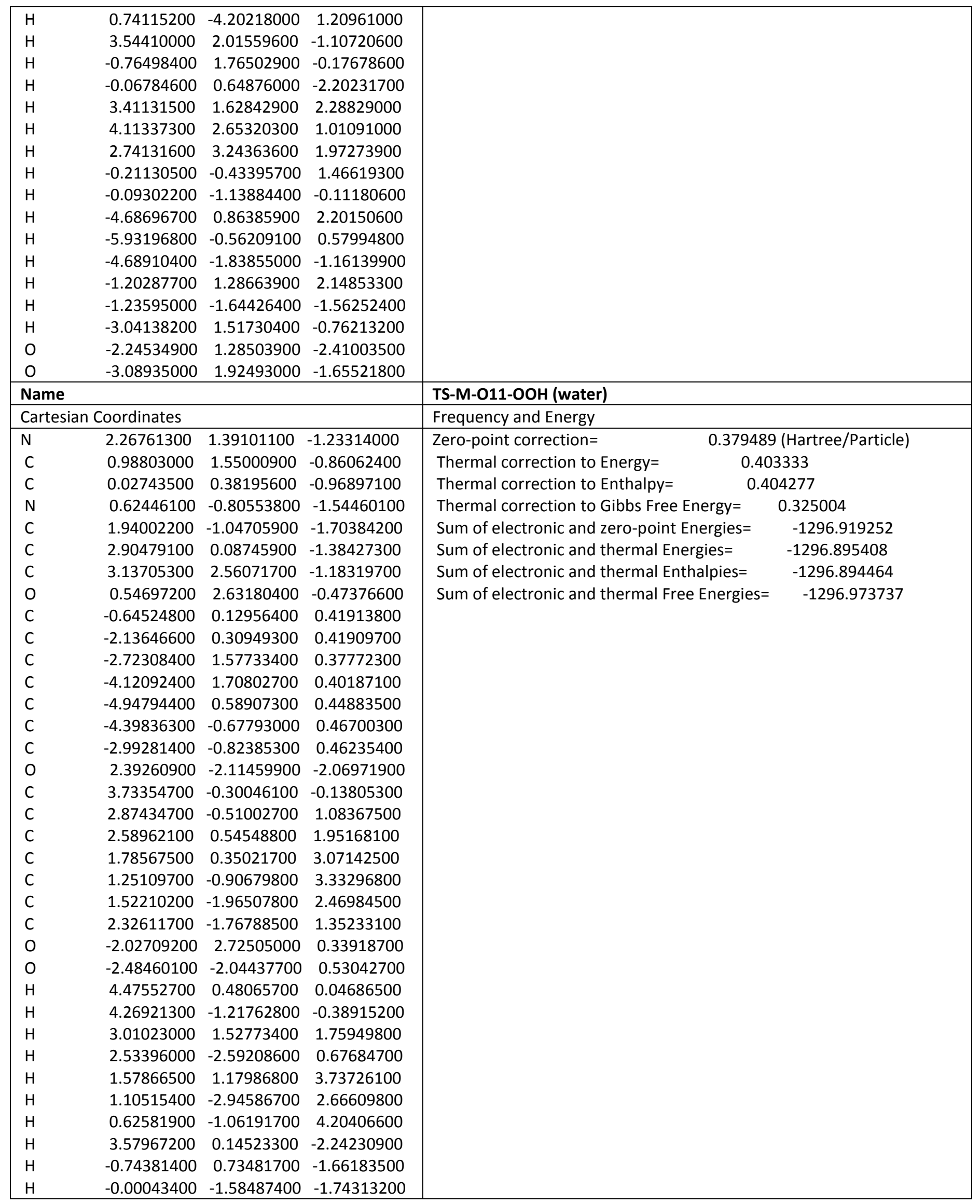




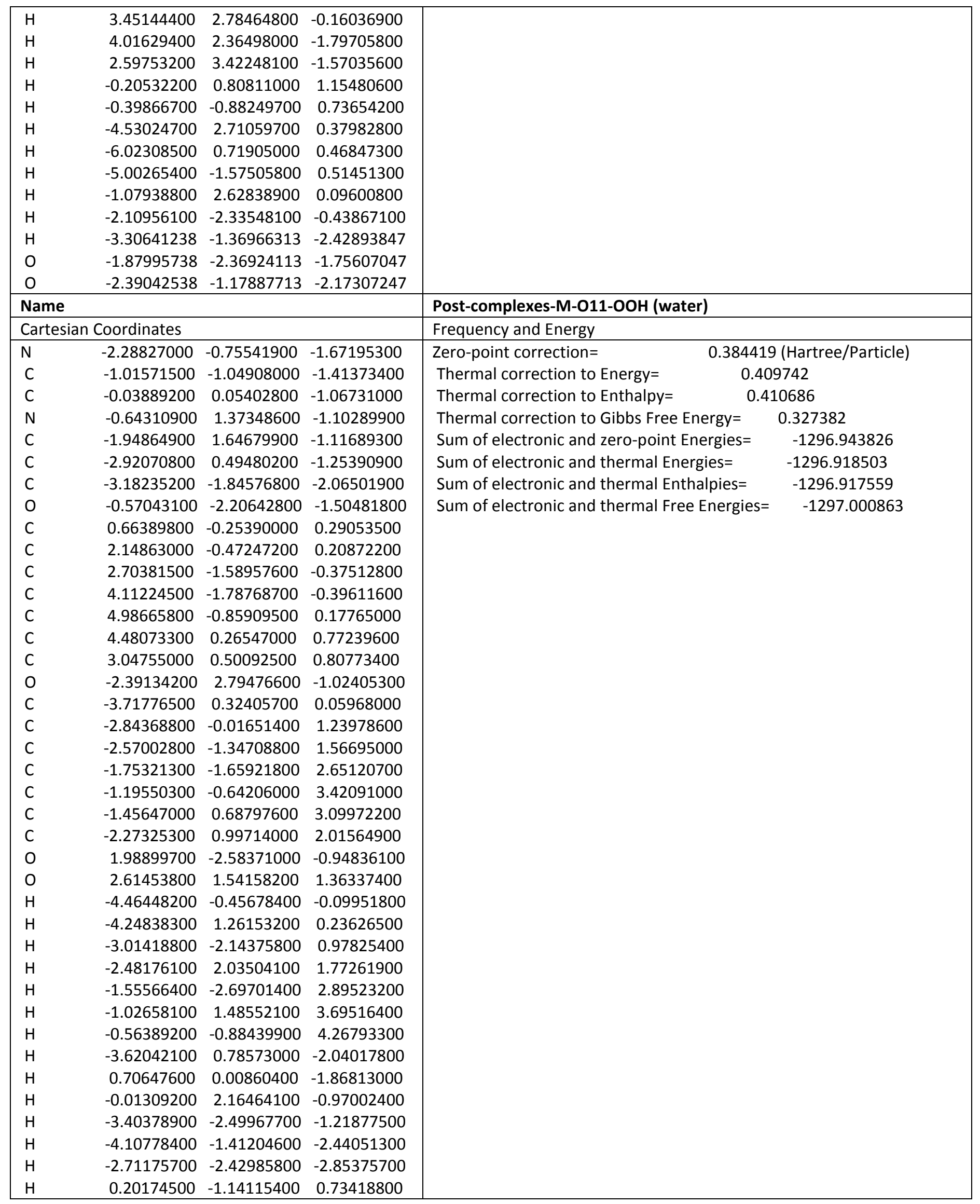




\begin{tabular}{|lrrr|l|}
\hline$H$ & 0.46767800 & 0.57801500 & 0.96529000 & \\
$H$ & 4.48790700 & -2.68806000 & -0.86757700 & \\
$H$ & 6.05358600 & -1.04048500 & 0.14686000 & \\
$H$ & 5.11518700 & 1.01352200 & 1.23226400 & \\
$H$ & 1.02796400 & -2.37614200 & -1.07425100 & \\
$H$ & 1.87665700 & 2.70234400 & 0.22870600 & \\
$H$ & 3.21428600 & 2.68654600 & -1.63546900 & \\
O & 1.68366800 & 3.15210900 & -0.62140500 & \\
O & 2.32659700 & 2.30530400 & -1.56444000 & \\
\hline
\end{tabular}




\section{References}

(1) Evans, M. G.; Polanyi, M. Some Applications of the Transition State Method to the Calculation of Reaction Velocities, Especially in Solution. Trans. Faraday Soc. 1935, 31, 875-894.

(2) Eyring, H. The Activated Complex in Chemical Reactions. J. Chem. Phys. 1935, 3, 107-115.

(3) Truhlar, D. G.; Hase, W. L.; Hynes, J. T. Current Status of Transition-State Theory. J. Phys. Chem. 1983, 87, 2664-2682.

(4) Furuncuoglu, T.; Ugur, I.; Degirmenci, I.; Aviyente, V. Role of Chain Transfer Agents in Free Radical Polymerization Kinetics. Macromolecules 2010, 43, 1823-1835.

(5) Vélez, E.; Quijano, J.; Notario, R.; Pabón, E.; Murillo, J.; Leal, J.; Zapata, E.; Alarcón, G. A Computational Study of Stereospecifity in the Thermal Elimination Reaction of Menthyl Benzoate in the Gas Phase. J. Phys. Org. Chem. 2009, 22, 971-977.

(6) Pollak, E.; Pechukas, P. Symmetry Numbers, Not Statistical Factors, Should Be Used in Absolute Rate Theory and in Broensted Relations. J. Am. Chem. Soc. 1978, 100, 2984-2991.

(7) Fernández-Ramos, A.; Ellingson, B. A.; Meana-Pañeda, R.; Marques, J. M.; Truhlar, D. G. Symmetry Numbers and Chemical Reaction Rates. Theor. Chem. Acc. 2007, 118, 813-826.

(8) Eckart, C. The Penetration of a Potential Barrier by Electrons. Phy. Rev. 1930, 35, 1303.

(9) Marcus, R. A. Chemical and Electrochemical Electron-Transfer Theory. Annu. Rev. Phys. Chem. 1964, 15, 155-196.

(10) Marcus, R. A. Electron Transfer Reactions in Chemistry. Theory and Experiment. Rev. Mod. Phys. 1993, 65, 599.

(11) Lu, Y.; Wang, A.; Shi, P.; Zhang, H. A Theoretical Study on the Antioxidant Activity of Piceatannol and Isorhapontigenin Scavenging Nitric Oxide and Nitrogen Dioxide Radicals. PloS one 2017, 12, e0169773.

(12) Lu, Y.; Wang, A.; Shi, P.; Zhang, H.; Li, Z. Quantum Chemical Study on the Antioxidation Mechanism of Piceatannol and Isorhapontigenin toward Hydroxyl and Hydroperoxyl Radicals. PloS one 2015, 10, e0133259.

(13) Nelsen, S. F.; Blackstock, S. C.; Kim, Y. Estimation of Inner Shell Marcus Terms for Amino Nitrogen Compounds by Molecular Orbital Calculations. J. Am. Chem. Soc. 1987, 109, 677-682.

(14) Nelsen, S. F.; Weaver, M. N.; Luo, Y.; Pladziewicz, J. R.; Ausman, L. K.; Jentzsch, T. L.; O'Konek, J. J. Estimation of Electronic Coupling for Intermolecular Electron Transfer from Cross-Reaction Data. J. Phys. Chem. A 2006, 110, 11665-11676.

(15) Galano, A.; Alvarez-Idaboy, J. R. A Computational Methodology for Accurate Predictions of Rate Constants in Solution: Application to the Assessment of Primary Antioxidant Activity. J. Comput. Chem. 2013, 34, 2430-2445.

(16) Collins, F. C.; Kimball, G. E. Diffusion-Controlled Reaction Rates. J. Colloid Sci. 1949, 4, 425-437.

(17) Von Smoluchowski, M. Mathematical Theory of the Kinetics of the Coagulation of Colloidal Solutions. Z. Phys. Chem 1917, 92, 129-68.

(18) Truhlar, D. G. Nearly Encounter-Controlled Reactions: The Equivalence of the Steady-State and Diffusional Viewpoints. J. Chem. Educ. 1985, 62, 104.

(19) Einstein, A. On the Motion of Small Particles Suspended in Liquids at Rest Required by the MolecularKinetic Theory of Heat. Ann. Phys. 1905, 17, 549-560.

(20) Stokes, G. G., Mathematical and Physical Papers. University Press: Cambridge, 1905. 
(21) Galano, A.; Raúl Alvarez-Idaboy, J. Computational Strategies for Predicting Free Radical Scavengers' Protection against Oxidative Stress: Where Are We and What Might Follow? Int. J. Quantum Chem. 2019, 119, e25665.

(22) Vo, Q. V.; Gon, T. V.; Bay, M. V.; Mechler, A. The Antioxidant Activity of Monosubstituted Indolinonic Hydroxylamines: A Thermodynamic and Kinetic Study. J. Phys. Chem. B 2019, 123, 10672-10679.

(23) Vo, Q. V.; Mechler, A. In Silico Study of the Radical Scavenging Activities of Natural Indole-3-Carbinols. J. Chem. Inf. Model. 2020, 60, 316-321.

(24) Okuno, Y. Theoretical Investigation of the Mechanism of the Baeyer-Villiger Reaction in Nonpolar Solvents. Chem.: Eur. J. 1997, 3, 212-218.

(25) Benson, S., The Foundations of Chemical Kinetics: . Malabar, Florida, 1982.

(26) Iuga, C.; Alvarez-Idaboy, J. R.; Vivier-Bunge, A. Ros Initiated Oxidation of Dopamine under Oxidative Stress Conditions in Aqueous and Lipidic Environments. J. Phys. Chem. B 2011, 115, 12234-12246.

(27) Alvarez-Idaboy, J. R.; Reyes, L.; Mora-Diez, N. The Mechanism of the Baeyer-Villiger Rearrangement: Quantum Chemistry and Tst Study Supported by Experimental Kinetic Data. Org. Biomol. Chem. 2007, 5, 3682-3689.

(28) Le, T. H.; Tran, T. T.; Huynh, L. K. Identification of Hindered Internal Rotational Mode for Complex Chemical Species: A Data Mining Approach with Multivariate Logistic Regression Model. Chemom. Intell. Lab. Syst. 2018, 172, 10-16. 